\title{
Anti-EGFR targeted therapy delivered before versus during radiotherapy in locoregionally advanced nasopharyngeal carcinoma: a big-data, intelligence platform-based analysis
}

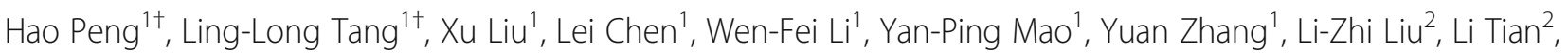
Ying Guo ${ }^{3}$, Ying Sun ${ }^{1}$ and Jun $\mathrm{Ma}^{1 *}$

\begin{abstract}
Background: Little is known about the prognostic difference of anti-EGFR therapy, cetuximab (CTX) or nimotuzumab (NTZ), concurrently with induction chemotherapy (IC, investigational arm) or RT (control arm) for patients with locoregionally advanced nasopharyngeal carcinoma (LA-NPC). We conducted this retrospective study to address this.

Methods: We identified 296 patients with newly diagnosed LA-NPC at Sun Yat-Sen University Cancer Center between January 2012 and May 2015. Patients were treated by IC with CCRT or RT and CTX/NTZ was delivered during IC or radiotherapy. Survival outcomes and toxicities between different arms were compared.

Results: In total, there were 149 patients in the investigational arm and 147 in control arm. The 3-year diseasefree survival, overall survival, distant metastasis-free survival and locoregional relapse-free survival rates for investigational arm vs. control arm were $84.3 \%$ vs. $74.3 \%$ ( $P=0.027), 94.0 \%$ vs. $92.1 \%(P=0.673), 88.0 \%$ vs. $81.8 \%(P=0.147)$ and $93.3 \%$ vs. 88.0\% ( $P=0.093)$. Multivariate analysis revealed patients in the control arm achieved significantly worse diseasefree survival $(H R, 1.497 ; 95 \% \mathrm{Cl}, 1.016-2.206 ; P=0.026)$ compared with those in the investigational arm; however, no significant difference was identified for other endpoints. Patients in the investigational arm experienced more grade $3-4$ skin reaction $(15.4 \%$ vs. $2.0 \%, P<0.001)$ and mucositis $(10.1 \%$ vs. $3.4 \%, P=0.022)$ during induction phase, but less skin reaction ( $5.4 \%$ vs. $25.9 \%, P<0.001)$ and mucositis ( $24.8 \%$ vs. $36.7 \%, P=0.026)$ during RT.
\end{abstract}

Conclusions: Our findings suggested that CTX/NTZ concurrently with IC may be a more effective and promising strategy for patients with LA-NPC receiving intensity-modulated radiotherapy.

Keywords: Nasopharyngeal carcinoma, Induction chemotherapy, Cetuximab, Nimotuzumab, Intensity-modulated radiotherapy, Prognosis

\footnotetext{
* Correspondence: majun2@mail.sysu.edu.cn

${ }^{\dagger}$ Equal contributors

'Department of Radiation Oncology, Sun Yat-sen University Cancer Center,

State Key Laboratory of Oncology in Southern China, Collaborative

Innovation Center for Cancer Medicine, 651 Dongfeng Road East,

Guangzhou 510060, People's Republic of China

Full list of author information is available at the end of the article
}

(c) The Author(s). 2018 Open Access This article is distributed under the terms of the Creative Commons Attribution 4.0 International License (http://creativecommons.org/licenses/by/4.0/), which permits unrestricted use, distribution, and reproduction in any medium, provided you give appropriate credit to the original author(s) and the source, provide a link to the Creative Commons license, and indicate if changes were made. The Creative Commons Public Domain Dedication waiver (http://creativecommons.org/publicdomain/zero/1.0/) applies to the data made available in this article, unless otherwise stated. 


\section{Background}

Nasopharyngeal carcinoma (NPC) is a special type of head and neck malignancy for its extremely unbalanced geographic distribution and treatment modality. There are 86, 700 new cases reported worldwide in 2012 with the highest incidence in South China [1]. Unlike other head and neck cancers, radiotherapy (RT) is the primary and only cure for non-disseminated disease as a result of the anatomic constrain and sensitivity to radiation. Control of early stage disease with RT alone or chemoradiation is usually excellent; however, management of locoregionally advanced NPC (LA-NPC) still remain unsatisfactory, with a 5-year overall survival (OS) of 67$77 \%$ [2]. Unfortunately, more than $70 \%$ of newly cases were locoregionally advanced disease at initial diagnosis [3]. Currently, concurrent chemo-radiation (CCRT) is the main standard care for LA-NPC. Although local and regional control has improved greatly, distant metastasis rates after treatment remain high and is the main source of treatment failure [4]. Therefore, identification of novel and effective therapeutic strategies is urgent and crucial for clinicians.

Epidermal growth factor receptor (EGFR), a transmembrane protein highly expressed in most human epithelial malignancies [5], is a promising therapeutic target in oncology for its correlation with aggressive phenotype, treatment resistance and poor prognosis $[6,7]$. EGFR is also highly expressed in NPC [8] and numerous studies have evaluated the efficacy of anti-EGFR targeted therapy [9-15]. Cetuximab (CTX) or nimotuzumab (NTZ) (anti-EGFR monoclonal antibodies) concurrently with RT could achieved comparable outcomes compared with standard cisplatin-RT $[12,14]$. When delivered during CCRT, different results were produced. You et al. and Xia et al. revealed CTX/NTZ additional to CCRT was more effective than CCRT alone $[11,13]$ while $\mathrm{Li}$ et al. did not identified any difference [10]. Regardless of the controversial efficacy, CTX/NTZ significantly increased acute mucositis and acneiform rash during RT $[10,12]$, resulting in poor quality of life or even disruption of RT. It seems that anti-EGFR therapy concurrent with RT may not be the best choice.

Induction chemotherapy (IC), given before RT, has been proven a promising treatment in LA-NPC for its satisfactory compliance and efficacy in reducing distant metastasis [16-19]. Possibly, CTX/NTZ in combination with IC may further reduce distant metastasis and improve survival outcomes. Notably, all abovementioned studies focus on the concurrent phase and no relative study to date has been carried out to assess the value of anti-EGFR therapy concurrently with IC. Given this concern, we initiated this retrospective study to evaluate the efficacy and toxicity difference of CTX/NTZ concurrently with IC or RT for LA-NPC.

\section{Methods}

Study patient

We identified 14,684 patients with newly diagnosed NPC on the big-data, intelligence database platform (YiduCloud Technology Ltd., Beijing, China) at Sun Yat-sen University Cancer center between January 2012 and May 2015. Inclusion criteria for this study were as follow: (i) stage III-IVB disease; (ii) age $\geq 18$ years; (iii) karnofsky performance score (KPS) $\geq 70$; (iv) did not have prior malignancies; (v) receiving IC followed by CCRT or RT alone; (vi) concurrent chemotherapy, if have, should be single-agent cisplatin; (vii) receiving intensity-modulated radiotherapy (IMRT).

\section{Pre-treatment staging work-up}

Conventional staging workup in our center included physical examination of head and neck, direct nasopharyngoscopy, chest radiography or computed tomography (CT), magnetic resonance imaging (MRI) of head and neck, abdominal sonography, whole-body bone scan and blood profile. Positron emission tomography (PET)-CT would also be recommended for patients with advanced N (N2-3) category. Magnetic resonance (MR) or CT scans of patients were reviewed separately by two radiologists employed at our center with more than 10-year experience, and any discrepancy was resolved by consensus. Tumor stage was grouped according to the 7th edition of the International Union against Cancer/American Joint Committee on Cancer (UICC/AJCC) system [20].

\section{Treatment}

All patients received radical IMRT at our center using the simultaneous integrated boost (SIB) technique as previously described. $[18,21]$ Briefly, prescribed radiation dose were 66-70 Gy at 2.12-2.27 Gy/fraction to the planning target volume (PTV) of nasopharyngeal gross tumor volume (GTV), 64-70 Gy to the PTV of GTV of metastatic lymph nodes, 60-63 Gy to the PTV of high-risk clinical target volume, and 50-56 Gy to the PTV of low-risk clinical target volume.

IC consisted of docetaxel $\left(75 \mathrm{mg} / \mathrm{m}^{2} \mathrm{~d} 1\right)$ with cisplatin (75 mg/m $\left.{ }^{2} \mathrm{~d} 1\right)(\mathrm{TP})$, fluorouracil $\left(1000 \mathrm{mg} / \mathrm{m}^{2} \mathrm{~d} 1-\mathrm{d} 5\right)$ with cisplatin $\left(80 \mathrm{mg} / \mathrm{m}^{2} \mathrm{~d} 1\right)(\mathrm{PF})$, or docetaxel $\left(60 \mathrm{mg} / \mathrm{m}^{2} \mathrm{~d} 1\right)$ plus cisplatin $\left(60 \mathrm{mg} / \mathrm{m}^{2} \mathrm{~d} 1\right)$ with fluorouracil $(600-$ $750 \mathrm{mg} / \mathrm{m}^{2} \mathrm{~d} 1-\mathrm{d} 5$ ) (TPF) every three weeks for 2-4 cycles. Concurrent chemotherapy was tri-weekly cisplatin (80$\left.100 \mathrm{mg} / \mathrm{m}^{2}\right)$ or weekly cisplatin $\left(30-40 \mathrm{mg} / \mathrm{m}^{2}\right)$.

CTX was delivered at a dose of $400 \mathrm{mg} / \mathrm{m}^{2}$ and NTZ was administered at a dose of $200 \mathrm{mg}$ concurrently with IC (investigation arm) every three weeks. For patients receiving anti-EGFR therapy during RT (control arm), NTZ was administered at a dose of $200 \mathrm{mg}$ weekly, and CTX was delivered at an initial dose of $400 \mathrm{mg} / \mathrm{m}^{2}$ followed by $250 \mathrm{mg} / \mathrm{m}^{2}$ weekly [13, 14]. Detailed information on treatment was presented in Additional file 1: Method S1. 


\section{Clinical endpoints and statistical analysis}

The first endpoint is disease-free survival (DFS) defined as the time from diagnosis to disease progression or death from any cause. Other endpoints included OS (time from diagnosis to death from any cause), distant metastasis-free survival (DMFS, time from diagnosis to first distant metastasis) and locoregional relapse-free survival (LRRFS, time from diagnosis to local or regional recurrence or both). Tumor response to IC was evaluated based on Response Evaluation Criteria in Solid Tumors [22]. Acute toxicities during IC were graded according to the Common Terminology Criteria for Adverse Events (version 3.0).

The Chi-square test were adopted to compare categorical variables and Mann-Whitney test for continuous variables. Survival outcomes were calculated using Kaplan-Meier method and compared by log-rank test. Multivariate cox proportional hazards model was used to estimate hazard ratios (HRs), 95\% confidence intervals (CIs) and independent prognostic factors. All tests were two-sided; $P<0.05$ was considered significant. Stata Statistical Package 12 (StataCorp LP, College Station, TX, USA) was used for all analyses.

\section{Results}

\section{Patient baseline characteristics}

Flow chart of patient inclusion was presented in Fig. 1. In total, we identified 2999 patients and an eventual 296 patients were eligible for this study with 149 in the investigation arm and 147 in control arm. Baseline characteristics were summarized in Table 1 . The median age for the whole cohort is 42 years (range, 18-73 years), and male-to-female ratio is 3.9:1. Host, tumor and treatment related factors were well balanced between the investigational arm and control arm. Moreover, patients in these two groups had similar pre-treatment imaging stage workups (Additional file 2: Table S1).

Among the investigational arm, 56 (37.6\%) received CTX and the remaining $93(62.4 \%)$ patients received NTZ. Within the control arm, 25 (17.0\%) patients received CTX and 122 (83.0\%) received NTZ. Obviously, more patients received NTZ during RT than that during IC $(P<0.001)$. Detailed information on dose and cycle of CTX/NTZ in each arm was shown in Additional file 3: Table S2. Undoubtedly, patients in the control arm received more cycles of CTX/NTZ. No dose reduction occurred in the two arms.

\section{Short-term efficacy after IC}

Sixteen patients with N0 category were not available for regional response evaluation, with 7 (4.7\%) in the investigational arm and $9(6.1 \%)$ in the control arm. After the completion of IC, 17 (11.4\%), 121 (81.2\%) and 11 (7.4\%) in the investigational arm, and 13 (8.8\%), $118(80.3 \%)$ and $16(10.9 \%)$ in the control arm achieved complete response (CR), partial response (PR) and stable disease (SD), respectively $(P=0.476)$. No patient had progression disease (PD) in both arms. Additional file 4: Table S3 detailed the information on tumor response.

\section{Long-term outcome analysis}

Up to the last visit (September 30, 2017), the median follow-up duration is 42.0 months (range 1.27-64.8). Among the whole cohort, the overall rates of locoregional and distant failures were $11.1 \%(33 / 296)$ and $15.9 \%$ (47/296), respectively. In detail, $26(17.4 \%)$ in the investigational arm and $42(28.6 \%)$ in control arm experienced treatment failure $(P=0.023)$. Additionally, $14(9.4 \%)$ and $16(10.9 \%)$ in the investigational and control arms died $(P=0.671)$. Three-year DFS, OS, DMFS and LRRFS rates for the whole cohort were $79.3 \%, 93.1 \%, 84.9 \%$ and 90 . $6 \%$, respectively.

The 3-year DFS, OS, DMFS and LRRFS rates for investigational arm vs. control arm were $84.3 \%$ vs. $74.3 \%$ $(P=0.027), 94.0 \%$ vs. $92.1 \%(P=0.673), 88.0 \%$ vs. $81.8 \%$ $(P=0.147)$ and $93.3 \%$ vs. $88.0 \%(P=0.093$, Fig. 2$)$. After adjusting for various prognostic factors, patients in the control arm achieved significantly worse DFS (HR, 1.497; 95\% CI, 1.016-2.206; $P=0.026$ ) compared with those in the investigational arm; however, no significant difference was identified between the two arms in terms of OS (HR, 0.994; 95\% CI, 0.466-2.122; $P=0.988$ ), DMFS (HR, 1.409; 95\% CI, 0.779-2.549; $P=0.251$ ) and LRRFS (HR, 1.805; 95\% CI, 0.883-3.686; $P=0.105$; Table 2).

\section{Grade 3-4 toxicities}

Acute toxicity profiles during IC and RT were evaluated between the two groups and results were presented in Table 3. Generally, grade 3-4 toxic events were comparable between the investigational and control arms $(58.4 \%$ vs. $58.5 \%, P=0.984)$. However, patients in the investigational arm experienced more grade $3-4$ skin reaction ( $15.4 \%$ vs. $2.0 \%, P<0.001)$ and mucositis $(10.1 \%$ vs. $3.4 \%$, $P=0.022)$ during induction phase, but less skin reaction (5.4\% vs. $25.9 \%, P<0.001)$ and mucositis $(24.8 \%$ vs. 36 . $7 \%, P=0.026)$ during RT compared with patients in the control arm. Haematological and gastrointestinal adverse events were similar between the two groups (all rates, $P>0.005)$.

\section{Discussion}

Managing advanced disease has always been a tough challenge not only in NPC management but also in many other cancers since prognosis of this subgroup is usually poor. Therefore, identification and establishment of novel and effective treatment is urgent and necessary. As far as we know, our study is the first one to compare the efficacy and safety of anti-EGFR therapy (CTX or 


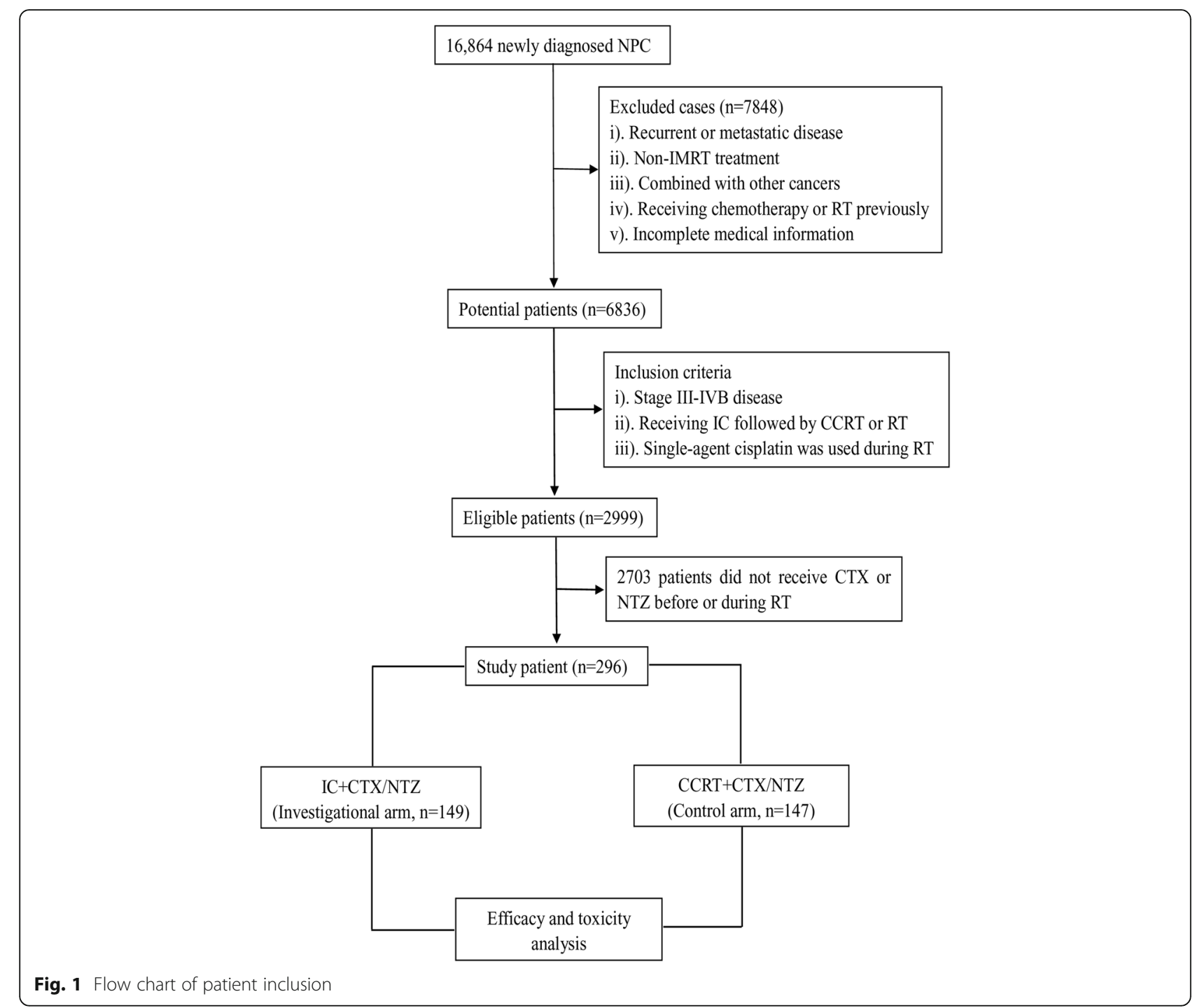

NTZ) concurrently with IC or RT in LA-NPC treated by IMRT. We found that CTX/NTZ delivered during IC could produce significantly better DFS than administered during RT while no significant difference was achieved with regard to OS, DMFS and LRRFS.

With the wide application of IMRT in NPC, local and regional control has improved greatly and distant metastasis has become the main failure pattern $[4,23]$. Although CCRT is effective, it may be not powerful enough to reduce distant metastasis for LA-NPC [24]. Additional cycles of chemotherapy like IC to CCRT is warranted. Although IC followed by CCRT has achieved excellent efficacy $[16,17,19]$, further improvement of prognosis is still needed. Therefore, novel and effective treatment strategies should be identified.

EGFR on tumor cells has been established as a factor predicting treatment resistance and poor prognosis $[6,7]$, making anti-EGFR a potential and promising treatment strategy. Antitumor efficacy of CTX in combination with conventional chemotherapy has been proven in various EGFR-expressing malignancies like colorectal cancer, head and neck cancers and recurrent NPC [25-27]. In recurrent or metastatic head and neck squamous cell carcinoma (HNSCC), CTX combined with fluorouracil-cisplatin chemotherapy achieved significantly better DFS and OS compared with fluorouracil-cisplatin alone when given as the first-line therapy [28]. It seems that CTX could overcome resistance to previously administered chemotherapy and thereby improved survival outcomes [26]. You et al. [13] and $\mathrm{Li}$ et al. [10] enhanced the treatment intensity during concurrent phase by adding CTX/NTZ to standard concomitant cisplatin. However, the efficacy may be unsatisfactory and adverse events significantly increased $[10,12-14]$. It's likely that concurrent administration of anti-EGFR therapy with cisplatin is a feasible strategy but not the best. These evidence reminded us that 
Table 1 Baseline characteristics of the 296 patients with stage III-IVB nasopharyngeal carcinoma

\begin{tabular}{llll}
\hline Characteristics & $\begin{array}{l}\text { Investigational arm } \\
(N=149, \%)\end{array}$ & $\begin{array}{l}\text { Control arm } \\
(N=147, \%)\end{array}$ & $P$-value \\
\hline $\begin{array}{l}\text { Gender } \\
\text { Male }\end{array}$ & $116(77.9)$ & $120(81.6)$ & 0.419 \\
$\quad$ Female & $33(22.1)$ & $27(18.4)$ & \\
Age (years) & & & 0.544 \\
$\quad$ Median (IQR) & $42(36-51)$ & $43(36-52)$ & \\
Smoking & & $62(42.2)$ & 0.491 \\
Yes & $57(38.3)$ & $85(57.8)$ & \\
No & $92(61.7)$ & & 0.095 \\
Drinking & & $19(12.9)$ & \\
Yes & $30(20.1)$ & $128(87.1)$ & \\
No & $119(79.9)$ & &
\end{tabular}

Family history of cancer

$\begin{array}{lll}\text { Yes } & 47(31.5) & 43(29.3) \\ \text { No } & 102(68.5) & 104(70.7)\end{array}$

$\mathrm{LDH}(\mathrm{U} / \mathrm{L})$

\begin{tabular}{|c|c|c|c|}
\hline Median (IQR) & $175(154-216)$ & $184(161-215)$ & \\
\hline re-DNA ${ }^{b}$ & & & 0.957 \\
\hline Median (IQR) & $6880(106-77,950)$ & $7445(494-52,050)$ & \\
\hline category $^{c}$ & & & 0.112 \\
\hline $\mathrm{T} 1$ & $6(4.0)$ & $1(0.7)$ & \\
\hline T2 & $7(4.7)$ & $14(9.5)$ & \\
\hline T3 & $81(54.4)$ & 77 (52.4) & \\
\hline T4 & $55(36.9)$ & $55(37.4)$ & \\
\hline
\end{tabular}

N category ${ }^{c}$

$\begin{array}{ll}\text { N0 } & 7(4.7) \\ \text { N1 } & 49(32.9) \\ \text { N2 } & 61(40.9) \\ \text { N3 } & 32(21.5) \\ \text { Overall stage } & \end{array}$

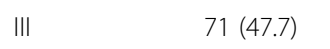

IVA-B $78(52.3)$

TPF regimen (cycles)

$\begin{array}{lll}\text { Two } & 15(30.0) & 14(41.6) \\ \text { Three } & 33(66.0) & 18(50.3) \\ \text { Four } & 2(4.0) & 2(8.1) \\ \text { PF regimen } & \text { (cycles) } & \\ \text { Two } & 42(93.3) & 51(96.3) \\ \text { Three } & 3(6.7) & 6(2.9) \\ \text { Four } & 0(0) & 0(0)\end{array}$

TP regimen (cycles)

$\begin{array}{ll}\text { Two } & 43(79.6) \\ \text { Three } & 7(13.0)\end{array}$

$45(59.9)$

$10(34.0)$
Table 1 Baseline characteristics of the 296 patients with stage III-IVB nasopharyngeal carcinoma (Continued)

\begin{tabular}{cclc}
\hline Characteristics & $\begin{array}{l}\text { Investigational arm } \\
(N=149, \%)\end{array}$ & $\begin{array}{l}\text { Control arm } \\
(N=147, \%)\end{array}$ & $P$-value \\
\hline Four & $4(7.4)$ & $1(6.1)$ & 0.964 \\
Concurrent chemotherapy & & \\
Yes & $130(87.2)$ & $128(87.1)$ & \\
No & $19(12.8)$ & $19(12.9)$ & \\
\hline
\end{tabular}

IC induction chemotherapy, IQR interquartile, $L D H$ lactate dehydrogenase; Pre$D N A$ pre-treatment Epstein-Barr virus DNA, TPF docetaxel plus cisplatin with fluorouracil, PF cisplatin with fluorouracil, TP docetaxel with cisplatin

${ }^{a} P$-values were calculated using Chi-square test for categorical variables and Mann-Whitney test for continuous variables

'Three patients in the control arm did not have this data

'According to the 7th edition of the International Union against Cancer/

American Joint Committee on Cancer (UICC/AJCC) system

CTX/NTZ in combination with IC may be a preferable choice. In our present study, we confirmed this view as patients in the investigational arm achieved significantly better DFS. Our findings provided a new insight in managing LA-NPC by enhancing the treatment intensity during IC.

Reasons contributing to the significantly difference of DFS were complicated. First, treatment intensity during IC was improved by adding CTX/NTZ which could help to further eradicate micro-metastasis lesions prior to RT and thereby reduce treatment failure events. Second, patients receiving CTX/NTZ during RT experienced more severe toxicities than those not. Consequently, these patients had poor quality of life during RT which could adversely affect prognosis [29, 30]. Furthermore, some patients even suffered RT interruption due to severe skin reaction or mucositis. Undoubtedly, the prolonged treatment time had a negative impact on prognosis [31, 32].

Notably, univariate and multivariate analysis only identified the significant difference between the two arms for DFS. As indicated by the survival curves, patients in the investigational arm also achieved better DMFS and LRRFS compared with patients in the control arm, although the difference was not significant. The main reason should be attributed to the small sample size which was not statistically powerful enough to identify the difference. Possibly, the improved DFS should originate from DMFS and LRRFS together. Due to the insufficient follow-up duration, no significant difference was achieved on OS although more patients in the control arm experienced treatment failure. Therefore, future studies with larger sample and longer follow-up duration are warranted to validate our results. Moreover, some other factors like program death ligand 1 (PD-L1) on tumor cells, human papillomavirus (HPV) status and lifetime cigarette smoking (pack-years) may also play important roles in prognosis and have an effect on the insignificant OS. However, these factors were not routinely evaluated in our center. Further studies were needed to evaluate these factors. 


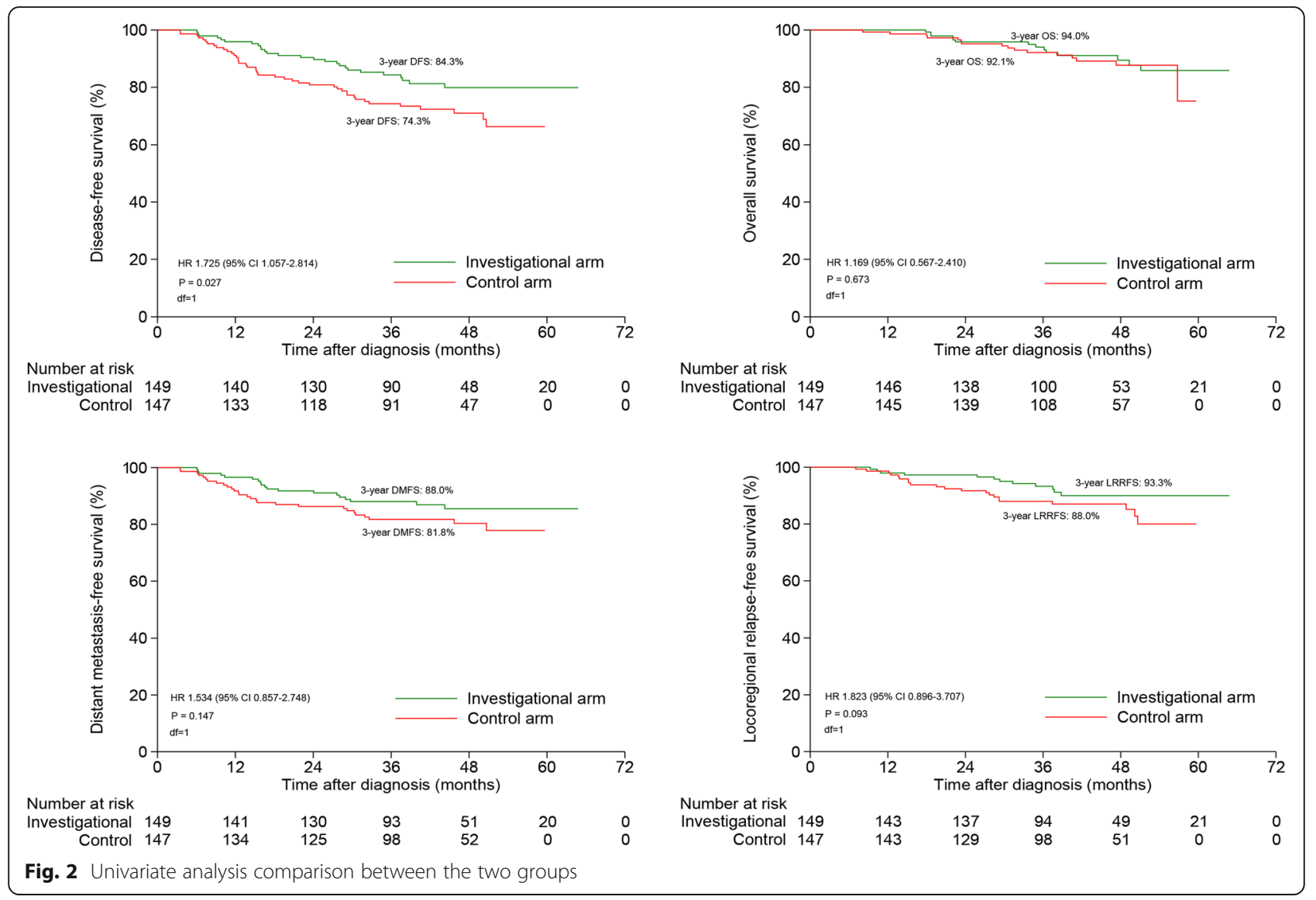

Table 2 Multivariate regression analysis for prognostic factors

\begin{tabular}{llll}
\hline Variable & $H R$ & $95 \% \mathrm{Cl}$ & \\
\hline Disease-free survival & & & \\
N category (N2-3 vs. N0-1) & 2.512 & $1.388-4.548$ \\
Overall stage (IV vs. III) & 1.757 & $1.051-2.939$ & 0.002 \\
Treatment group (Control vs. investigational) & 1.497 & $1.016-2.206$ & \\
Overall survival & & & \\
Overall stage (IV vs. III) & 4.995 & $1.907-13.083$ \\
Treatment group (Control vs. investigational) & 0.994 & $0.466-2.122$ \\
Distant metastasis-free survival & & & 0.026 \\
N category (N2-3 vs. N0-1) & 2.791 & $1.344-5.793$ \\
Treatment group (Control vs. investigational) & 1.409 & $0.779-2.549$ \\
Locoregional relapse-free survival & & & 0.988 \\
IC regimen (TPF vs. PF) & 0.204 & $0.059-0.707$ \\
IC regimen (TPF vs. TP) & 0.823 & $0.398-1.701$ \\
Treatment group (Control vs. investigational) & 1.805 & $0.883-3.686$ \\
\hline
\end{tabular}

HR hazard ratio, Cl confidence interval, IC induction chemotherapy, TPF docetaxel plus cisplatin with fluorouracil, PF cisplatin with fluorouracil, TP docetaxel with cisplatin, $L D H$ lactate dehydrogenase

${ }^{a}$ Multivariate $P$-values were calculated by Cox proportional hazard regression model with backward elimination for the following prognostic factors: gender (female vs. male), age (> 42y vs. $\leq 42 y$ ), smoking (yes vs. no), drinking (yes vs. no), family history of cancer (yes vs. no), LDH ( $>245$ vs. $\leq 245$ U/L), IC regimen (TPF vs. PF, TPF vs. TP), concurrent chemotherapy (yes vs. no), T category (T3-4 vs. T1-2), N category (N2-3 vs. N0-1), Overall stage (IV vs. III) and treatment group (control vs. investigational) 
Table 3 Grade 3-4 acute toxicity profiles during induction chemotherapy and radiotherapy

\begin{tabular}{llll}
\hline Grade 3-4 toxicity & $\begin{array}{l}\text { Investigational arm } \\
(N=149, \%)\end{array}$ & $\begin{array}{l}\text { Control arm } \\
(N=147, \%)\end{array}$ & $P$ value \\
\hline Any & $87(58.4 \%)$ & $86(58.5 \%)$ & 0.984 \\
Induction phase & & & \\
Leucopenia & $36(24.2)$ & $27(18.4)$ & 0.223 \\
Neutropenia & $61(40.9)$ & $45(30.6)$ & 0.064 \\
Anaemia & $2(1.3)$ & $2(1.4)$ & 0.989 \\
Thrombocytopenia & $5(3.4)$ & $1(0.7)$ & 0.102 \\
Liver function & $3(2.0)$ & $2(1.4)$ & 0.663 \\
Renal function & $0(0)$ & $0(0)$ & 1.000 \\
Skin reaction & $23(15.4)$ & $3(2.0)$ & $<0.001$ \\
Mucositis & $15(10.1)$ & $5(3.4)$ & 0.022 \\
Nausea & $3(2.0)$ & $2(1.4)$ & 0.663 \\
Vomiting & $8(5.4)$ & $12(8.2)$ & 0.338 \\
Diarrhoea & $3(2.0)$ & $1(0.7)$ & 0.321 \\
Concurrent phase & & & \\
Leucopenia & $31(20.8)$ & $40(27.2)$ & 0.197 \\
Neutropenia & $19(12.8)$ & $24(16.3)$ & 0.383 \\
Anaemia & $11(7.4)$ & $15(10.2)$ & 0.391 \\
Thrombocytopenia & $13(8.7)$ & $8(5.4)$ & 0.271 \\
Liver function & $3(2.0)$ & $5(3.4)$ & 0.462 \\
Renal function & $0(0)$ & $2(1.4)$ & 0.246 \\
Skin reaction & $8(5.4)$ & $38(25.9)$ & $<0.001$ \\
Mucositis & $37(24.8)$ & $54(36.7)$ & 0.026 \\
Nausea & $14(9.4)$ & $18(12.2)$ & 0.430 \\
Vomiting & $20(13.4)$ & $22.4)$ & 0.704 \\
Diarrhoea & $2(1.3)$ & 0.244 \\
\hline
\end{tabular}

${ }^{\mathrm{a}} P$-values were calculated by Chi-square test or Fisher exact test

Generally, severe toxicities during RT in our study were similar as the findings in previous studies [10, 12-14]. Overall grade 3-4 toxic events were comparable between the two arms; however, anti-EGFR therapy related toxicities like skin reaction and mucositis significantly varied between the investigational and control arms during IC and RT. In detail, patients in the investigational arm experienced more grade $3-4$ skin reaction and mucositis during IC but less during RT. Undoubtedly, CTX/NTZ could aggravate radiation-induced skin and oral mucositis. Another reason for the difference may be that the total dose used in induction phase is less than that used in concurrent phase. As both arms had similar chemotherapy intensity, severe heamatological and gastrointestinal events were parallel.

In our study, patients in the investigational arm had two main advantages compared with those in control arm. First, patients experienced significantly less anti-EGFR therapy related severe toxicities. Therefore, patients had better quality of life during treatment. Second, cycles of CTX/ NTZ used during induction phase were usually less than that in concurrent phase. Hence, cost of anti-EGFR therapy is also less. However, limitations of this study should also be acknowledged. Our study is retrospective and the sample size may be small, meaning that potential bias existed and the power of identify difference may be insufficient. Moreover, the follow-up duration may be insufficient. Consequently, we set DFS as the first endpoint to address this. Furthermore, the cycles of IC were not uniform. In light of previous evidence, we recruited patients receiving at least two cycles because two cycles could produce the similar survival outcomes as three or more cycles [21]. Importantly, we balanced this factor between the two groups. The median age of included patients is younger than that in other reports, which should be attributed to the small sample size.

\section{Conclusion}

In summary, CTX/NTZ concurrently with IC could achieved better survival outcomes and less severe toxicities compared with CTX/NTZ concurrently with RT in LA-NPC treated by IMRT. Our study provided a new insight into managing LA-NPC for clinicians. However, findings of present study need to be validated in prospective studies.

\section{Additional files}

Additional file 1: Supplementary Method. (DOCX 17 kb)

Additional file 2: Table S1. Imaging methods for the 296 patients in the two groups. (DOCX $14 \mathrm{~kb}$ )

Additional file 3: Table S2. Cycle and total dose of CTX and NTZ for in each arm. (DOCX $13 \mathrm{~kb}$ )

Additional file 4: Table S3. Tumor response after IC between investigational and control arms. (DOCX $14 \mathrm{~kb}$ )

\section{Abbreviations}

CCRT: concurrent chemo-radiation; Cl: confidence interval; CT: computed tomography; CTX: Cetuximab; DFS: disease-free survival; DMFS: distant metastasis-free survival; EGFR: Epidermal growth factor receptor; GTV: gross tumor volume; HNSCC: head and neck squamous cell carcinoma; HR: hazard ratio; IC: Induction chemotherapy; IMRT: receiving intensity-modulated radiotherapy; KPS: karnofsky performance score; LA-NPC: locoregionally advanced nasopharyngeal carcinoma; LRRFS: locoregional relapse-free survival; MRI: magnetic resonance imaging; NPC: Nasopharyngeal carcinoma; NTZ: nimotuzumab; OS: overall survival; PET: positron emission tomography; PF: fluorouracil with cisplatin; PTV: planning target volume; RT: radiotherapy; SIB: simultaneous integrated boost; TP: docetaxel with cisplatin;

TPF: docetaxel plus cisplatin with fluorouracil; UICC/AJCC: International Union against Cancer/American Joint Committee on Cancer

\section{Acknowledgements}

We sincerely thanked Dr. Wei Liang and Dr. Lei Shi (YiduCloud Technology Ltd., Beijing, China) for kindly providing technical support in searching data on the big-data intelligence platform. 


\section{Funding}

This work was supported by grants from the National Science \& Technology Pillar Program during the Twelfth Five-year Plan Period (2014BAI09B10), the Natural Science Foundation of Guangdong Province (2017A030312003), the National Natural Science Foundation of China (81572658), the Program of Introducing Talents of Discipline to Universities (B14035), and the Innovation Team Development Plan of the Ministry of Education (No. IRT_17R110).

\section{Availability of data and materials}

The datasets generated and analyzed during the current study are deposited in the Research Data Deposit (RDDA2017000416, http://www.researchdata.org.cn/ ), and available from the corresponding author.

\section{Authors' contributions}

HP, LLT, and JM contributed to study design and conception. HP, LZL, LT, YZ, $\mathrm{XL}$ and $Y P M$ contributed to data acquisition. HP, LC, JM, and $Y G$ analyzed and interpreted the data. HP, WFL, and $Y Z$ contributed to manuscript revision. YPM and YS contributed to quality control and review of the data and manuscript. All authors have read and approved the final version of the submitted manuscript.

\section{Ethics approval and consent to participate}

Written Informed consent was obtained from all patients before treatment and this study was approved by the institutional research ethics committee of Sun Yat-sen University Cancer Center.

\section{Consent for publication}

Not applicable.

\section{Competing interests}

The authors declare that they have no competing interests.

\section{Publisher's Note}

Springer Nature remains neutral with regard to jurisdictional claims in published maps and institutional affiliations.

\section{Author details \\ ${ }^{1}$ Department of Radiation Oncology, Sun Yat-sen University Cancer Center, State Key Laboratory of Oncology in Southern China, Collaborative Innovation Center for Cancer Medicine, 651 Dongfeng Road East, Guangzhou 510060, People's Republic of China. ${ }^{2}$ Imaging Diagnosis and Interventional Center, Sun Yat-sen University Cancer Center, State Key Laboratory of Oncology in Southern China, Collaborative Innovation Center for Cancer Medicine, Guangzhou, People's Republic of China. ${ }^{3}$ Department of Clinical Trials Center, Sun Yat-sen University Cancer Center, State Key Laboratory of Oncology in Southern China, Collaborative Innovation Center for Cancer Medicine, Guangzhou, People's Republic of China.}

Received: 14 December 2017 Accepted: 20 March 2018

Published online: 27 March 2018

\section{References}

1. Torre LA, Bray F, Siegel RL, Ferlay J, Lortet-Tieulent J, Jemal A. Global cancer statistics, 2012. CA Cancer J Clin. 2015;65:87-108

2. Yi JL, Gao L, Huang XD, et al. Nasopharyngeal carcinoma treated by radical radiotherapy alone: ten-year experience of a single institution. Int J Radiat Oncol Biol Phys. 2006;65:161-8.

3. Mao YP, Xie FY, Liu LZ, et al. Re-evaluation of 6th edition of AJCC staging system for nasopharyngeal carcinoma and proposed improvement based on magnetic resonance imaging. Int J Radiat Oncol Biol Phys. 2009;73:1326-34.

4. Lai SZ, Li WF, Chen L, et al. How does intensity-modulated radiotherapy versus conventional two-dimensional radiotherapy influence the treatment results in nasopharyngeal carcinoma patients? Int J Radiat Oncol Biol Phys. 2011;80:661-8

5. Zhang $\mathrm{H}$, Berezov $\mathrm{A}$, Wang $\mathrm{Q}$, et al. ErbB receptors: from oncogenes to targeted cancer therapies. J Clin Invest. 2007;117:2051-8.

6. Ciardiello F, Tortora GA. Novel approach in the treatment of cancer: targeting the epidermal growth factor receptor. Clin Cancer Res. 2001;7:2958-70

7. Mendelsohn J. Targeting the epidermal growth factor receptor for cancer therapy. J Clin Oncol. 2002;20(18 Suppl):1S-13S.
8. Ma BB, Poon TC, To KF, et al. Prognostic significance of tumor angiogenesis, Ki 67, p53 oncoprotein, epidermal growth factor receptor and HER2 receptor protein expression in undifferentiated nasopharyngeal carcinomaa prospective study. Head Neck. 2003:25:864-72.

9. He X, Xu J, Guo W, et al. Cetuximab in combination with chemoradiation after induction chemotherapy of locoregionally advanced nasopharyngeal carcinoma: preliminary results. Future Oncol. 2013;9:1459-67.

10. Li Y, Chen QY, Tang LQ, et al. Concurrent chemoradiotherapy with or without cetuximab for stage II to IVb nasopharyngeal carcinoma: a casecontrol study. BMC Cancer. 2017;17:567.

11. Xia WX, Liang H, LV X, et al. Combining cetuximab with chemoradiotherapy in patients with locally advanced nasopharyngeal carcinoma: a propensity score analysis. Oral Oncol. 2017;67:167-74.

12. Xu T, Liu Y, Dou S, Li F, Guan X, Zhu G. Weekly cetuximab concurrent with IMRT aggravated radiation-induced oral mucositis in locally advanced nasopharyngeal carcinoma: results of a randomized phase II study. Oral Oncol. 2015:51:875-9.

13. You R, Hua YJ, Liu YP, et al. Concurrent Chemoradiotherapy with or without anti-EGFR-targeted treatment for stage II-IVb nasopharyngeal carcinoma: retrospective analysis with a large cohort and long follow-up. Theranostics. 2017:7:2314-24.

14. You R, Sun R, Hua YJ, et al. Cetuximab or nimotuzumab plus intensitymodulated radiotherapy versus cisplatin plus intensity-modulated radiotherapy for stage II-IVb nasopharyngeal carcinoma. Int J Cancer. 2017; 141:1265-76.

15. Huang JF, Zhang FZ, Zou QZ, et al. Induction chemotherapy followed by concurrent chemoradiation and nimotuzumab for locoregionally advanced nasopharyngeal carcinoma: preliminary results from a phase II clinical trial. Oncotarget. 2017:8:2457-65.

16. Cao SM, Yang Q, Guo L, et al. Neoadjuvant chemotherapy followed by concurrent chemoradiotherapy versus concurrent chemoradiotherapy alone in locoregionally advanced nasopharyngeal carcinoma: a phase III multicentre randomised controlled trial. Eur J Cancer. 2017;75:14-23.

17. Hui EP, Ma BB, Leung SF, et al. Randomized phase II trial of concurrent cisplatin-radiotherapy with or without neoadjuvant docetaxel and cisplatin in advanced nasopharyngeal carcinoma. J Clin Oncol. 2009:27:242-9.

18. Peng H, Chen L, Zhang J, et al. Induction chemotherapy improved longterm outcomes of patients with Locoregionally advanced nasopharyngeal carcinoma: a propensity matched analysis of 5-year survival outcomes in the era of intensity-modulated radiotherapy. J Cancer. 2017:8:371-7.

19. Sun Y, Li WF, Chen NY, et al. Induction chemotherapy plus concurrent chemoradiotherapy versus concurrent chemoradiotherapy alone in locoregionally advanced nasopharyngeal carcinoma: a phase 3, multicentre, randomised controlled trial. Lancet Oncol. 2016;17:1509-20.

20. Edge SB, Compton CC. The American joint committee on Cancer: the 7th edition of the AJCC cancer staging manual and the future of TNM. Ann Surg Oncol. 2010;17:1471-4.

21. Peng $H$, Chen L, Li WF, et al. Optimize the cycle of neoadjuvant chemotherapy for locoregionally advanced nasopharyngeal carcinoma treated with intensity-modulated radiotherapy: a propensity score matching analysis. Oral Oncol. 2016:62:78-84.

22. Therasse P, Arbuck SG, Eisenhauer EA, et al. New guidelines to evaluate the response to treatment in solid tumors. European Organization for Research and Treatment of Cancer, National Cancer Institute of the United States, National Cancer Institute of Canada. J Natl Cancer Inst. 2000;92:205-16.

23. Sun X, Su S, Chen C, et al. Long-term outcomes of intensity-modulated radiotherapy for 868 patients with nasopharyngeal carcinoma: an analysis of survival and treatment toxicities. Radiother Oncol. 2014;110:398-403.

24. Lin JC, Liang WM, Jan JS, Jiang RS, Lin AC. Another way to estimate outcome of advanced nasopharyngeal carcinoma-is concurrent chemoradiotherapy adequate? Int J Radiat Oncol Biol Phys. 2004;60:156-64.

25. Chan AT, Hsu MM, Goh BC, et al. Multicenter, phase II study of cetuximab in combination with carboplatin in patients with recurrent or metastatic nasopharyngeal carcinoma. J Clin Oncol. 2005;23:3568-76.

26. Cunningham D, Humblet $Y$, Siena $S$, et al. Cetuximab monotherapy and cetuximab plus irinotecan in irinotecan-refractory metastatic colorectal cancer. N Engl J Med. 2004;351:337-45.

27. Pfister DG, Su YB, Kraus DH, et al. Concurrent cetuximab, cisplatin, and concomitant boost radiotherapy for locoregionally advanced, squamous cell head and neck cancer: a pilot phase II study of a new combined-modality paradigm. J Clin Oncol. 2006;24:1072-8. 
28. Vermorken JB, Mesia R, Rivera F, et al. Platinum-based chemotherapy plus cetuximab in head and neck cancer. N Engl J Med. 2008;359:1116-27.

29. Fang FM, Chiu HC, Kuo WR, et al. Health-related quality of life for nasopharyngeal carcinoma patients with cancer-free survival after treatment. Int J Radiat Oncol Biol Phys. 2002;53:959-68.

30. Fang FM, Tsai WL, Chien CY, et al. Pretreatment quality of life as a predictor of distant metastasis and survival for patients with nasopharyngeal carcinoma. J Clin Oncol. 2010;28:4384-9.

31. Kwong DL, Sham JS, Chua DT, Choy DT, Au GK, Wu PM. The effect of interruptions and prolonged treatment time in radiotherapy for nasopharyngeal carcinoma. Int J Radiat Oncol Biol Phys. 1997;39:703-10.

32. Li PJ, Jin T, Luo DH, et al. Effect of prolonged radiotherapy treatment time on survival outcomes after intensity-modulated radiation therapy in nasopharyngeal carcinoma. PLoS One. 2015;10:e0141332.

\section{Submit your next manuscript to BioMed Central} and we will help you at every step:

- We accept pre-submission inquiries

- Our selector tool helps you to find the most relevant journal

- We provide round the clock customer support

- Convenient online submission

- Thorough peer review

- Inclusion in PubMed and all major indexing services

- Maximum visibility for your research

Submit your manuscript at www.biomedcentral.com/submit 